\title{
Phytoconstituents and Proximate Composition of Clerodendrum Colebrookianum Walp.: A Widely Used Anti High Blood Pressure Medicinal Food Plant in Eastern Himalayas
}

\section{Temin Payum*}

\section{Temin Payum*}

Jawaharlal Nehru College, Pasighat, Department of Botany, Arunachal Pradesh 791103, INDIA.

\section{Correspondence \\ Dr. Temin Payum}

Assistant Professor, Department of Botany, Jawaharlal Nehru College, Pasighat,

East Siang District, P.O: Hilltop 791103

Arunachal Pradesh, INDIA.

E-mail: teminpayum519@gmail.com

History

- Submission Date: 17-07-2020;

- Review completed: 02-09-2020;

- Accepted Date: 22-09-2020

DOI : 10.5530/pj.2020.12.210

Article Available online

http://www.phcogj.com/v12/i6s

\section{Copyright}

(C) 2020 Phcogj.Com. This is an openaccess article distributed under the terms of the Creative Commons Attribution 4.0 International license.

\begin{abstract}
Clerodendrum colebrookianumWalp.is a medicinal food plant widely used in the North East India. The herb is used as vegetable as well as medicine to control high blood pressure. Minerals and Proximate compositions in a food is vital for the proper growth and development of a healthy body and secondary metabolites included in diet act as a nutraceuticals thus help in fighting various health problems. The present study was carried out to discourse the Phytoconstituents, Proximate composition and Minerals of the nutraceutical herb, Clerodendrum colebrookianum. Methanol extract of sample was subjected to GCMS to profile the Phyoconstituents while Standard methods including AOAC was followed to study proximate and minerals of the sample under studied. Moisture content was $77.90 \%$, carbohydrate $4.28 \%$, $2.36 \%$ crude protein, $4.21 \%$ crude fibre and $0.35 \%$ crude fat respectively. The minerals concentrations are $0.215 \mathrm{mg}$ of $\mathrm{Fe} / \mathrm{g}, 0.105 \mathrm{mg} / \mathrm{g}$ of $\mathrm{Mn}, 0.0425 \mathrm{mg}$ of $\mathrm{Cu} / \mathrm{g}, 0.056 \mathrm{mg} \mathrm{Zn/g}$, $2.55 \mathrm{mg}$ of Mangnesium $/ \mathrm{g}, 4.3 \mathrm{mg}$ of $\mathrm{Na} / \mathrm{g}$ and $24.5 \mathrm{mg}$ of $\mathrm{K}$ per gram of sample. A total of eleven compounds are recorded to be useful for high blood pressure problem and as many as other twenty useful phytoconstutuents were recorded from the sample including antioxidant, anti-uric acid formation, anti-tumour, bioabiability of zinc etc. The present study advocates the traditional knowledge on the use of Clerodendrum colebrookianum as a remedy for high blood pressure problem.

Key words: Clerodendrum colebrookianum, North East India, Nutraceutical Herb, Phytoconstituents, Proximate, Minerals.
\end{abstract}

\section{INTRODUCTION}

Let food be your medicine, once said, Hippocrates ${ }^{1}$. Galen- "the father of observational medicine" believed that the fundamentals of good medicine lay in diet. And, such medicinal food concepts still observable in the indigenous food system practices among indigenous people in various pockets of the world. In the word of Etkin \& Ross ${ }^{2}$, wild plants that are retained in local food cultures are inseparable from traditional therapeutic systems. And, Pieroni and Price ${ }^{3}$ remarked that it is difficult to draw a line between food and medicine; food may be medicine and medicine may be food. And, In remote rural Villages, Wild edible plants are integral source of food, medicine, shelter and livelihood. Many of wild edible vegetables have medicinal property and can be used to treat common ailments; these are easily available, cheap and also excellent source of nutrients like proteins, carbohydrates, iron, essential minerals and other secondary metabolites. And, regular uses of these vegetables act as an alternative source of medicinal drugs along with nutritional benefits ${ }^{4}$. There is a vast cornucopia of herbs and foods which stimulate support and nourish our body system. Some have been used by different traditional systems of several countries and are now being evaluated by modern research ${ }^{5}$. Medicinal and aromatic plants represent an inexhaustible source of life saving drugs for the majority of the world's population ${ }^{6}$. The beneficial remedial effects of plant materials are mainly due to the mixture of substances called secondary metabolites of plants ${ }^{7}$.

Hypertension is one of the major chronic diseases, which affect people around the world. One approach to control hypertension is by using diet containing an adequate amount of phytochemicals; increased intake of phytochemicals is associated with decreased mortality rate from cardiovascular diseases, stroke and injuries secondary to hypertension ${ }^{8}$. Hypertension or high blood pressure accounts 9.4 million deaths all over the world every year; The use of plant origin natural compounds as cardio protective and antihypertensive agents rich in a variety of secondary metabolites such as flavonoids, alkaloids, tannins and terpenoids are helpful ${ }^{10}$. And many have turned their attention to the use of herbal medicines for hypertension treatment in country like Thailand ${ }^{10}$

And, Clerodendrum colebrookianium Walp.(fig.1.), a medicinal food plants widely used among Indigenous people of North East India as medicinal food to lower down the high blood pressure. Some workers have reported the traditional uses of this herb in high blood pressure related practices from the eastern Himalayan regions of India ${ }^{11-13}$. With the above backdrops, the present research has been designed to study the proximate composition, phytoconstituents and minerals present in C.colebrookianum.
Cite this article: Payum T. Phytoconstituents and Proximate Composition of Clerodendrum Colebrookianum Walp.: A Widely Used Anti High Blood Pressure Medicinal Food Plant in Eastern Himalayas. Pharmacogn J. 2020;12(6)Suppl:1534-40. 


\section{MATERIAL AND METHODS}

\section{Plant material}

Clerodendrum colebrookianum Walp.; a Verbanaceae family is a shrub of about $15 \mathrm{ft}$. high with disagreeable smell. Bark shining light grey; Leaves: $3.5-10$ by $2.5-8.5$ in., broad, ovate, acute, entire, membraneous, almost glabrous; lateral nerves 6-9 on either half; base shallow cordate; petiole 0.5-6.5 in. long with cluter of glands near the apex. Flower is white in broad terminal compact, corymb biform compound cymes; bract caduceus. Calyx: pubescent, often bearing a few glands; calyx: teeth short; Corolla: tube slender, 1-1.25 in. long; style exerted; Fruit: Bluish green to deep green when fully ripe, glossy, 0.3 in. across, globose, compressed above, of 4 duprels seated on an accrescent cup-shaped calyx about 0.4 in. Across ${ }^{14}$. The material was collected from Kitchen garden of Mrs Oki Paron of Pasighat Town of East Siang District of Arunachal Pradesh, India.

\section{Preparation of extract for GCMS}

The shoot was washed thoroughly in distilled water and dried in shade till the weight did not changed further and pulverized into powder using a mechanical grinder. $500 \mathrm{~g}$ of plant powder was soaked in ethanol (Merck) for 72 hours with intermittent shaking then filtered through Whatmann No. 41 filter paper and concentrated extract was obtained by using water bath.

\section{Proximate and minerals studies}

The shoot was washed thoroughly in distilled water and dried in shade till the weight did not changed further and pulverized into powder using a mechanical grinder, the powder was used in proximate analysis. For moisture study, fresh collected sample was used before drying. The Association of Official Analytical Chemists (AOAC, 1990) were used for the determination of ash, crude lipid, crude fibre, carbohydrate and mineral. Ash was determined in silica crucibles by inciration in a muffle furnace at $550^{\circ} \mathrm{C}$ for $5 \mathrm{hrs}$. Crude lipid was extracted by continuous soxlet methods (AOAC, 1990) with petroleum ether (b.p $40-60^{\circ} \mathrm{C}$ ). Crude fiber was estimated by acid-base digestion with $1.25 \% \mathrm{H}_{2} \mathrm{SO}_{4}$ and $1.25 \% \mathrm{NaOH}$ solution. Nitrogen was estimated by Kjeldelmethod with steam distillation and titrated with standard 0.01 M HCL solution. Crude protein was estimated by multiplying the sample per cent Nitrogen content by a factor 6.25 . (\% protein $=\%$ Nitrogen X 6.26). Carbohydrate was estimated by Anthrone Method with an ultravioletvisible (UV-Vis) spectrophotometer (Lamda-25, Perkin Elmer, Cambridge UK). Minerals were analysed from solution obtained when $1.0 \mathrm{~g}$ of the samples were digested with concentrated $10 \mathrm{ml}$ nitric acid and kept overnight and heated till fumes of $\mathrm{HNO}_{3}$ and allowed to cool and add $4 \mathrm{ml}$ concentrated perchloric acid and heated again till clear solution is obtained and filtered into $100 \mathrm{ml} \mathrm{ml} \mathrm{standard} \mathrm{flask} \mathrm{and}$ made to mark with distilled water and analysed in atomic absorption spectrophotometer (Buck scientific model 200A).

\section{GC-MS analysis environment}

Gas-Chromatography Mass Spectrometry (GC-MS) analysis of the ethanol extracts of C. colebrrokianium carried out in Shimadzu GCMSQP-2010 plus system. RTx-5 Sil MS column (30 m X 0.25 mm id X 0.25 film thickness) was used for the analysis. The operating conditions of the column were as follows: Oven temperature program from $80^{\circ} \mathrm{C}$ to $210^{\circ} \mathrm{C}$ at $4^{\circ} \mathrm{C} / \mathrm{min}$ withhold time of $2 \mathrm{~min}$ and from $210^{\circ} \mathrm{C}$ to $300^{\circ} \mathrm{C}$ at $15^{\circ} \mathrm{C} / \mathrm{min}$ withhold time of $5 \mathrm{~min}$, and the final temperature was kept for $20 \mathrm{~min}$. The injector temperature was maintained at $270^{\circ} \mathrm{C}$, the volume of injected sample was $0.3 \mu \mathrm{l}$; pressure $85.4 \mathrm{kPa}$, total flow $76.8 \mathrm{~mL} / \mathrm{min}$, column flow $1.21 \mathrm{~mL} / \mathrm{min}$, linear velocity $40.5 \mathrm{~cm} / \mathrm{sec}$, purge flow $3.0 \mathrm{~mL} / \mathrm{min}$, split ratio: 60.0 ; ion source temperature $230^{\circ} \mathrm{C}$; scan mass range of $\mathrm{m} / \mathrm{z} \quad 40-600$ and interface line temperature $280^{\circ} \mathrm{C}$.
The identification of compounds was performed by comparing their mass spectra with data from NIST 11 (National Institute of Standards and Technology, US) and WILEY 8.

\section{RESULTS AND DISCUSSION}

A total of forty four phytoconstituents were recorded form the shoot sample of C.colebrookianum; out of which most of the phytoconstituents are useful compounds for a healthy growth and development. $14.56 \%$ of peak area was 2,2-DIDEUTEROTRANS-1,3-DIHYDROXY-CYCLOPENTANE, it is CatecholO-Methyl-transferase-inhibitor, glucosyl-transferase-inhibitor, reverse ranscriptase inhibitor; ; another $14.02 \%$ peak area was 9,12-OCTADECADIENOIC ACID (Z,Z)- it Inhibit Production of Uric Acid, Increase Zinc Bioavailibility ${ }^{*}$ and Hepatoprotective ${ }^{16} ; 10.46 \%$ peak area was PENTADECANOIC ACID and it Increase Aromatic Amino Acid Decarboxylase Activity, Inhibit Production of Uric Acid, Increase Zinc*; $4.17 \%$ was Squalene, it is Monooxygenase-inhibitor*, antioxidant, antitumour ${ }^{17}$, it also Hepatoprotective, Hypolipidemic, hypoglycemic $^{16}$. 2,3-DIHYDRO-3,5-DIHYDROXY-6-METHYL4H-PYRAN-4-ONE with 1.56\%; which is Catechol-O-Methyltransferase-inhibitor, 11B-HSD-inhibitor, 5-HETE-INHIBITOR, Hepatoprotective* $; 8.06 \%$ of peak area was dl-.alpha.-Tocopherol, it is Antioxidant, 5-alpha- reductase-inhibitor, Tocopherol synergist, increase alpha-mannosidase activity ${ }^{*}$ Antihypertension ${ }^{17} ; 5.08 \%$ was (2E)-3,7,11,15-TETRAMETH YL-2-HEXADECEN-1-OL; it is Anticancer, Antidote(emetine), endocroneprotective, endocrine tonic $^{*}$; $1.14 \%$ peak was 9,12-OCTADECADIENAL, DIMETHYL ACETAL,it is Hepatoprotective ${ }^{16 ;} .2 .03 \%$ peak area was Octadecanoic acid, it Increase Aromatic Amino Acid Decarboxylase Activity, Inhibit Production of Uric Acid, Increase Zinc ${ }^{*}$ Hypocholoresterolemic ${ }^{16}$; 4.83\% was Stigmasterol, it also Antihypercholesterolemic, Antioxidant, antitumour $^{18,19} ; 0.29 \%$ was OCTADECANOIC ACID, ETHYL ESTER, it Increase Aromatic Amino Acid Decarboxylase Activity, Inhibit Production of Uric Acid, Increase Zinc ${ }^{*}$, Hypocholeterolemic ${ }^{16} ; 0.32 \%$ pear area was 14-.BETA.-H-PREGNA, it is Hepatoprotective, Histidine kinase-inhibitor, Hypercholesterolemic, hypoglycemic, hypolipemic, increase $\mathrm{T}$ helper*; $0.72 \%$ peak area was gamma.-Tocopherol, it is Antioxidant, Tocopherol synergist, PPAR-Gamma-Antagonist* ${ }^{*}$ Cardioprotective ${ }^{16} ; 2.10 \%$ peak area was 5 -Hydroxymethyl furfural, it is Aromatic ${ }^{15}$; $1.04 \%$ peak area was 3-[N'-(3H-Indol-3-ylmethylene)hydrazino]-5-methyl-[1,2,4]triazol-4-ylamine, it is Neurostimulant, Antitumor (Nasopharynx), Nephroprotective, Neurostimulant*; $0.08 \%$ peak area was 1,2-BENZENEDICARBOXYLIC ACID, DIETHYL ESTER, it Increase Aromatic Amino Acid Decarboxylase Activity, Inhibit Production of Uric Acid, Increase Zinc*; $0.32 \%$ peak area was .alpha-Terpineol, it is 5-alpha reductase-inhibitor, HIF1 alpha-inhibitor*; $0.12 \%$ peak area was TETRADECANOIC ACID, it Increase Aromatic Amino Acid Decarboxylase Activity, Inhibit Production of Uric Acid, Increase Zinc*; $0.18 \%$ peak area was 3-Chloropropionic acid, heptadecyl ester, it inhibit Production of Uric Acid, Increase Zinc ${ }^{*}$; 3.82\% peak area was 2,6,10-TRIMETHYL,14ETHYLENE-14-PENTADECNE， it is Antiproliferative ${ }^{16} ; 0.91 \%$ peak area was 3,7,11,15-TETRAMETHYLHEXADEC-2-EN-1$\mathrm{OL}$, it is Endoanesthetic, endoprotective*; $1.82 \%$ peak area was 2-HEXADECEN-1-OL, 3,7,11,15-TETRAMETHYL-, [R-[R*, $\left.\left.\mathrm{R}^{*}-(\mathrm{E})\right]\right]$, it is 5-Alpha-Reductase-Inhibitor, Benzodiazepine-Receptor Agonist, Endothelium-Derived Relaxing Factor Promoter; $2.00 \%$ peak area was HEXADECANOIC ACID, ETHYL ESTER, it Inhibit Production of Uric Acid, Increase Zinc*; 2.24\% peak area was ETHYL (9Z,12Z)9,12-OCTADECADIENOATE, it Increase zinc bioavaiablilty*; $1.14 \%$ peak area was (R)-(-)-14-Methyl-8-hexadecyn-1-ol, it is CatecholO-Methyl-Transferase-Inhibir, Free-Radical Scavenging, HIV-RTInhibitor, Radioprotective; $0.75 \%$ peak area was 10 -UNDECEN1-AL, 2-METHYL, it is Catechol-O-Methyl transferase inhibitor, 

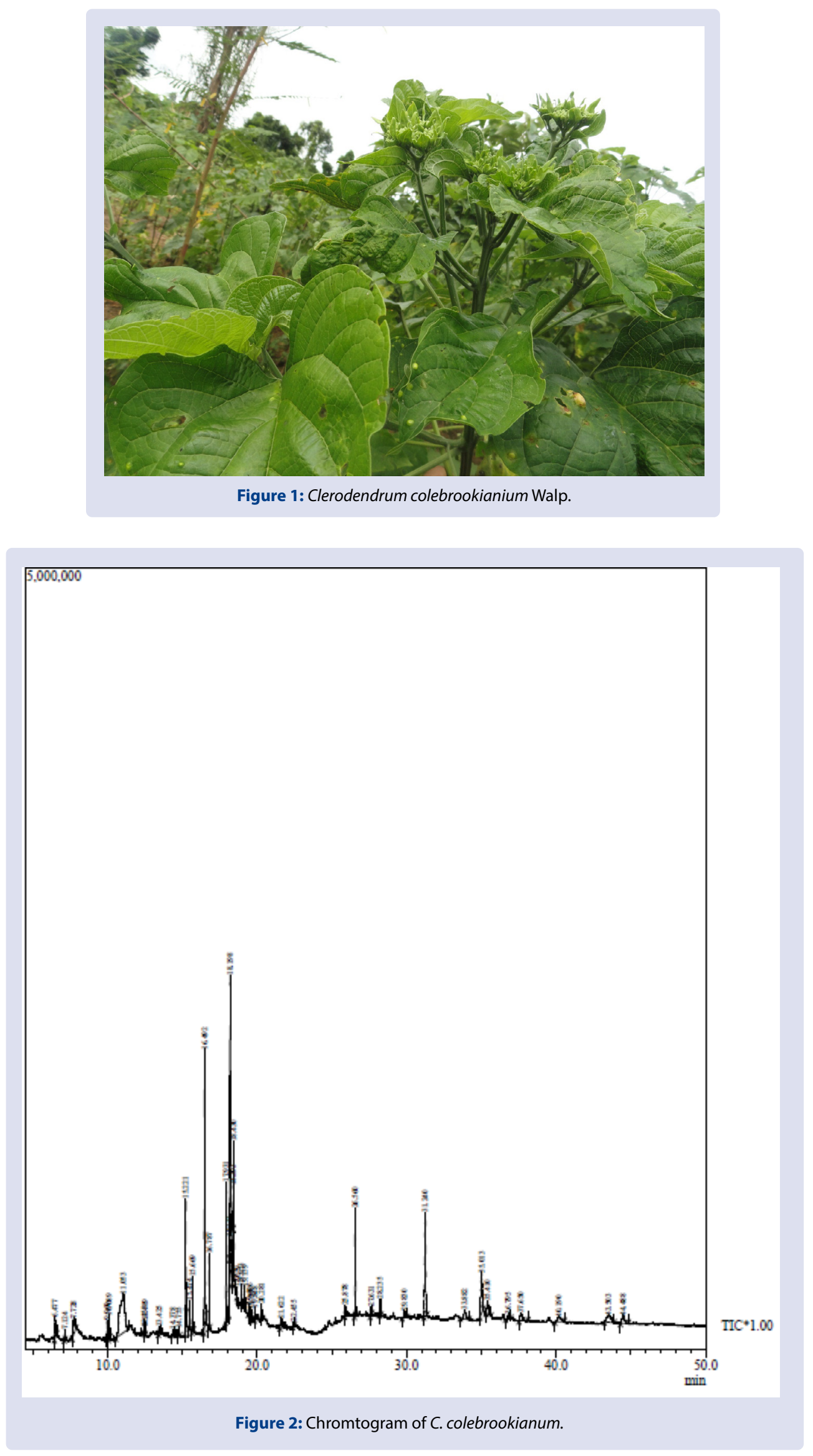
Payum et al:: Phytoconstituents and Proximate Composition of Clerodendrum Colebrookianum Walp.: A Widely Used Anti High Blood Pressure Medicinal Food Plant in Eastern Himalayas

Table 1: Compound table.

\begin{tabular}{|c|c|c|c|c|}
\hline Peak & R. time & Area & Area $\%$ & Compound name \\
\hline 1 & 6.477 & 590693 & 1.56 & 2,3-DIHYDRO-3,5-DIHYDROXY-6-METHYL-4H-PYRAN-4-ONE \\
\hline 2 & 7.124 & 97225 & 0.26 & 1-DODECENE \\
\hline 3 & 795253 & 795253 & 2.10 & 5-Hydroxymethylfurfural \\
\hline 4 & 9.960 & 165693 & 0.44 & 1-TRIDECENE \\
\hline 5 & 10.089 & 391550 & 1.04 & :3-[N'-(3H-Indol-3-ylmethylene)-hydrazino]-5-methyl-[1,2,4]triazol-4-ylamine \\
\hline 6 & 11.053 & 5506326 & 14.56 & :2,2-DIDEUTERO-TRANS-1,3-DIHYDROXY-CYCLOPENTANE \\
\hline 7 & 12.453 & 29341 & 0.08 & 1,2-BENZENEDICARBOXYLIC ACID, DIETHYL ESTER \\
\hline 8 & 12.489 & 63315 & 0.17 & 1-HEXADECENE \\
\hline 9 & 13.425 & 120208 & 0.32 & .alpha.-Terpineol \\
\hline 10 & 14.378 & 46366 & 0.12 & TETRADECANOIC ACID \\
\hline 11 & 14.755 & 68890 & 0.18 & 3-Chloropropionic acid, heptadecyl ester \\
\hline 12 & 15.221 & 1444503 & 3.82 & 2,6,10-TRIMETHYL,14-ETHYLENE-14-PENTADECNE \\
\hline 13 & 15.474 & 345089 & 0.91 & 3,7,11,15-TETRAMETHYLHEXADEC-2-EN-1-OL \\
\hline 14 & 15.669 & 689193 & 1.82 & 2-HEXADECEN-1-OL, 3,7,11,15-TETRAMETHYL-, [R-[R*, $\left.\left.\mathrm{R}^{\star}-(\mathrm{E})\right]\right]-$ \\
\hline 15 & 16.492 & 3957134 & 10.46 & PENTADECANOIC ACID \\
\hline 16 & 16.787 & 757965 & 2.00 & HEXADECANOIC ACID, ETHYL ESTER \\
\hline 17 & 17.931 & 1921800 & 5.08 & $\begin{array}{l}(2 \mathrm{E})-3,7,11,15 \text {-TETRAMETH } \\
\text { YL-2-HEXADECEN-1-OL }\end{array}$ \\
\hline 18 & 18.133 & 534632 & 1.41 & CYCLODECENE \\
\hline 19 & 18.198 & 5301217 & 14.02 & 9,12-OCTADECADIENOIC ACID (Z,Z)- \\
\hline 20 & 18.393 & 767186 & 2.03 & Octadecanoic acid \\
\hline 21 & 18.430 & 846091 & 2.24 & ETHYL (9Z,12Z)-9,12-OCTADECADIENOATE \\
\hline 22 & 18.659 & 109775 & 0.29 & OCTADECANOIC ACID, ETHYL ESTER \\
\hline 23 & 18.953 & 431547 & 1.14 & (R)-(-)-14-Methyl-8-hexadecyn-1-ol \\
\hline 24 & 19.159 & 430293 & 1.14 & 9,12-OCTADECADIENAL, DIMETHYL ACETAL \\
\hline 25 & 19.496 & 283923 & 0.75 & 10-UNDECEN-1-AL, 2-METHYL- \\
\hline 26 & 19.596 & 119959 & 0.32 & 14-.BETA.-H-PREGNA \\
\hline 27 & 19.848 & 152281 & 0.40 & 1-(1-HEPTADECYNYL)CYCLOPENTANOL \\
\hline 28 & 20.281 & 267469 & 0.71 & 5-(3,3-DIMETHYL-2-OXIRANYL)-3-METHYL-1-PENTANOL \\
\hline 29 & 21.622 & 349688 & 0.92 & $\begin{array}{l}\text { CYCLOOCTANEPENTANOIC ACID, 1-NITRO-.BETA.,2-DIOXO-, } \\
\text { METHYL ESTER }\end{array}$ \\
\hline 30 & 22.455 & 88747 & 0.23 & TETRADECANAL \\
\hline 31 & 25.878 & 158580 & 0.42 & Isolongifolene, $4,5,9,10$-dehydro- \\
\hline 32 & 26.560 & 1577068 & 4.17 & Squalene \\
\hline 33 & 27.631 & 148541 & 0.39 & Nonane, 3-methyl-5-propyl- \\
\hline 34 & 28.235 & 339316 & 0.90 & Tripropylsilyloxycyclobutane \\
\hline 35 & 29.830 & 271666 & 0.72 & .gamma.-Tocopherol \\
\hline 36 & 31.240 & 3046976 & 8.06 & dl-.alpha.-Tocopherol \\
\hline 37 & 33.882 & 486505 & 1.29 & 26,27-DINORCHOLESTA-5,22-DIEN-3-OL, (3.BETA.,22E)- \\
\hline 38 & 35.013 & 1826911 & 4.83 & Stigmasterol \\
\hline 39 & 35.410 & 644578 & 1.70 & gamma.-Sitosterol \\
\hline 40 & 36.795 & 131660 & 0.35 & Phytol, acetate \\
\hline 41 & 37.650 & 538888 & 1.42 & $\begin{array}{l}\text { 6.beta.Bicyclo[4.3.0]nonane, 5.beta.-iodomethyl-1.beta.-isopropenyl-4.alpha.,5. } \\
\text { alpha.-dimethyl-, }\end{array}$ \\
\hline 42 & 40.190 & 544847 & 1.44 & $\begin{array}{l}\text { 2,4A,8,8-TETRAMETHYL-DECAHYDRO-CYCLOPROPA[D]NAPHTHA- } \\
\text { LENE }\end{array}$ \\
\hline 43 & 43.503 & 894709 & 2.37 & Phytol, acetate \\
\hline \multirow[t]{2}{*}{44} & 44.488 & 541688 & 1.43 & Oxirane, hexadecyl- \\
\hline & & 37825285 & 100.00 & \\
\hline
\end{tabular}


Payum et al:: Phytoconstituents and Proximate Composition of Clerodendrum Colebrookianum Walp.: A Widely Used Anti High Blood Pressure Medicinal Food Plant in Eastern Himalayas

\section{Table 2: Useful Compound table.}

\begin{tabular}{|c|c|c|c|}
\hline Peak & Area \% & Compound name & Important of compound \\
\hline 1 & 1.56 & $\begin{array}{l}\text { 2,3-DIHYDRO-3,5-DIHYDROXY-6-METH- } \\
\text { YL-4H-PYRAN-4-ONE }\end{array}$ & $\begin{array}{l}\text { Catechol-O-Methyl-transferase-inhibitor, 11B-HSD-inhibitor, 5-HETE-INHIBITOR, } \\
\text { Anti-HIV-integrase, antidote(heavy metals)Hormone balancing, Hepatoprotective }\end{array}$ \\
\hline 2 & 2.10 & 5-Hydroxymethyl furfural & Aromatic $^{15}$ \\
\hline 3 & 1.04 & $\begin{array}{l}\text { 3-[N'-(3H-Indol-3-ylmethylene)-hydrazino]- } \\
\text { 5-methyl-[1,2,4]triazol-4-ylamine }\end{array}$ & Neurostimulant, Antitumor (Nasopharynx), Nephroprotective, Neurostimulant ${ }^{*}$ \\
\hline 4 & 14.56 & $\begin{array}{l}\text { 2,2-DIDEUTERO-TRANS-1,3-DIHYDROXY- } \\
\text { CYCLOPENTANE }\end{array}$ & Catechol-O-Methyl-transferase-inhibitor, reverse ranscriptase inhibitor, transdermal ${ }^{*}$ \\
\hline 5 & 0.08 & $\begin{array}{l}\text { 1,2-BENZENEDICARBOXYLIC ACID, DIETHYL } \\
\text { ESTER }\end{array}$ & $\begin{array}{l}\text { Increase Aromatic Amino Acid Decarboxylase Activity, Inhibit Production of Uric } \\
\text { Acid, Increase Zinc }{ }^{*}\end{array}$ \\
\hline 6 & 0.32 & .alpha.-Terpineol & $\begin{array}{l}\text { 5-alpha reductase-inhibitor, HIF1 alpha-inhibitor, IkappaB-alpha-phosphorylarion- } \\
\text { inhibitor, TNF-alpha inhibitor }\end{array}$ \\
\hline 7 & 0.12 & TETRADECANOIC ACID & $\begin{array}{l}\text { Increase Aromatic Amino Acid Decarboxylase Activity, Inhibit Production of Uric } \\
\text { Acid, Increase Zinc }\end{array}$ \\
\hline 8 & 0.18 & 3-Chloropropionic acid, heptadecyl ester & $\begin{array}{l}\text { Increase Aromatic Amino Acid Decarboxylase Activity, Inhibit Production of Uric } \\
\text { Acid, Increase Zinc }\end{array}$ \\
\hline 9 & 3.82 & $\begin{array}{l}\text { 2,6,10-TRIMETHYL,14-ETHYLENE-14-PEN- } \\
\text { TADECNE }\end{array}$ & Antiproliferative $e^{16}$ \\
\hline 10 & 0.91 & $\begin{array}{l}3,7,11,15 \text {-TETRAMETHYLHEXADEC-2-EN- } \\
1 \text {-OL }\end{array}$ & $\begin{array}{l}\text { Endoanesthetic, endoprotective, endothelium derived relaxing factor promoter, endo- } \\
\text { crine tonic }\end{array}$ \\
\hline 11 & 1.82 & $\begin{array}{l}\text { 2-HEXADECEN-1-OL, 3,7,11,15-TETRAMETH- } \\
\left.\left.\text { YL-, [R-[R*, } R^{*}-(\mathrm{E})\right]\right]-\end{array}$ & Respirasedative, 5-Alpha-Reductase-Inhibitor, Benzodiazepine-Receptor Agonist*. \\
\hline 12 & 10.46 & PENTADECANOIC ACID & $\begin{array}{l}\text { Increase Aromatic Amino Acid Decarboxylase Activity, Inhibit Production of Uric } \\
\text { Acid, Increase Zinc }\end{array}$ \\
\hline 13 & 2.00 & HEXADECANOIC ACID, ETHYL ESTER & $\begin{array}{l}\text { Increase Aromatic Amino Acid Decarboxylase Activity, Inhibit Production of Uric } \\
\text { Acid, Increase Zinc }\end{array}$ \\
\hline 14 & 5.08 & $\begin{array}{l}(2 \mathrm{E})-3,7,11,15-\mathrm{TETRAMETH} \\
\text { YL-2-HEXADECEN-1-OL }\end{array}$ & Anticancer()Esophagus, Antidote(emetine), endoanesthetic, endocroneprotective ${ }^{*}$ \\
\hline 15 & 14.02 & 9,12-OCTADECADIENOIC ACID (Z,Z)- & Inhibit Production of Uric Acid, Increase Zinc Bioavailibility; Hepatoprotective ${ }^{16}$ \\
\hline 16 & 2.03 & Octadecanoic acid & $\begin{array}{l}\text { Increase Aromatic Amino Acid Decarboxylase Activity, Inhibit Production of Uric } \\
\text { Acid, Increase Zinc }{ }^{*} \text { Hypocholoresterolemic }{ }^{16}\end{array}$ \\
\hline 17 & 2.24 & ETHYL (9Z,12Z)-9,12-OCTADECADIENOATE & Increase zinc bioavaiablilty \\
\hline 18 & 0.29 & OCTADECANOIC ACID, ETHYL ESTER & $\begin{array}{l}\text { Increase Aromatic Amino Acid Decarboxylase Activity, Inhibit Production of Uric } \\
\text { Acid, Increase Zinc } c^{*} \text { Hypocholeterolemic }{ }^{16} \text {. }\end{array}$ \\
\hline 18 & 1.14 & (R)-(-)-14-Methyl-8-hexadecyn-1-ol & $\begin{array}{l}\text { Catechol-O-Methyl-Transferase-Inhibitor, 5-Alpha-Reductase-Inhibitor, Free-Radical } \\
\text { Scavenging*. }\end{array}$ \\
\hline 19 & 1.14 & 9,12-OCTADECADIENAL, DIMETHYL ACETAL & Hepatoprotective ${ }^{16}$. \\
\hline 20 & 0.75 & 10-UNDECEN-1-AL, 2-METHYL- & Catechol-O-Methyl transferase inhibitor, detoxicant (alcohol) antidote(aluminum)* \\
\hline 21 & 0.32 & 14-.BETA.-H-PREGNA & $\begin{array}{l}\text { Hepatoprotective, Hypercholesterolemic, hypoglycemic, hypolipemic, increase T } \\
\text { helper }\end{array}$ \\
\hline 22 & 4.17 & Squalene & $\begin{array}{l}\text { Monooxygenase-inhibitor }{ }^{\star} \text {, antioxidant, antitumour }{ }^{17} \text {; Hepatoprotective, Hypolipid- } \\
\text { emic, hypoglycemic }{ }^{16} \text {. }\end{array}$ \\
\hline 23 & 0.72 & .gamma.-Tocopherol & Antioxidant, Tocopherol synergist, PPAR-Gamma-Antagonist* ${ }^{\star}$ Cardioprotective ${ }^{16}$. \\
\hline 24 & 8.06 & dl-.alpha.-Tocopherol & $\begin{array}{l}\text { Antioxidant, } 5 \text {-alpha- reductase-inhibitor, Tocopherol synergist, TNF-alpha inhibitor } \\
\text { Antihypertension }^{18} \text {. }\end{array}$ \\
\hline 25 & 4.83 & Stigmasterol & Antihypercholesterolemic, Antioxidant, antitumour ${ }^{19,20}$. \\
\hline 26 & 1.70 & gamma.-Sitosterol & $\begin{array}{l}\text { PPAR -Gamma-Antagonist }{ }^{*} \text { ( protection against obesity and related diseases such as } \\
\text { type } 2 \text { diabetes }{ }^{21} \text {. }\end{array}$ \\
\hline 27 & 1.44 & $\begin{array}{l}\text { 2,4A,8,8-TETRAMETHYL-DECAHYDRO- } \\
\text { CYCLOPROPA[D]NAPHTHALENE }\end{array}$ & Antimcrobial ${ }^{22}$. \\
\hline 28 & 2.37 & Phytol, acetate & Fragrance in cosmetic industry ${ }^{23}$. \\
\hline 29 & 1.43 & Oxirane, hexadecyl- & Antimicrobial ${ }^{24}$. \\
\hline
\end{tabular}

Dr.Duke's Phytochemical and Ethnobotanical Databases. 
Payum et al:: Phytoconstituents and Proximate Composition of Clerodendrum Colebrookianum Walp.: A Widely Used Anti High Blood Pressure Medicinal Food Plant in Eastern Himalayas

Table 3: Proximate composition of C. colebrookianum.

\begin{tabular}{|c|c|c|c|c|c|c|}
\hline \multicolumn{7}{|c|}{ Fresh weight basis; g/100g; $\mathrm{n}=3$. } \\
\hline Sample name & $\begin{array}{c}\text { Moisture } \\
(\%)\end{array}$ & Carbohydrate (\%) & Total ash (\%) & Crude protein (\%) & Crude fibre (\%) & Crude fat $(\%)$ \\
\hline C.colebrookianum & $77.90 \pm 0.08$ & $4.28 \pm 1.08$ & $11.15 \pm 0.63$ & $2.36 \pm 0.04$ & $4.21 .05 \pm 1.03$ & $0.35 \pm 0.03$ \\
\hline
\end{tabular}

Table 4: Mineral composition.

\begin{tabular}{|c|c|c|c|c|c|c|c|}
\hline \multirow{2}{*}{ Sample } & \multicolumn{7}{|c|}{ Concentration of Element (mg/g) } \\
\hline & $\mathrm{Fe}$ & $\mathrm{Mn}$ & $\mathrm{Cu}$ & $\mathrm{Zn}$ & $\mathrm{Mg}$ & $\mathrm{Na}$ & $\mathrm{K}$ \\
\hline C. colebrookianum & 0.215 & 0.105 & 0.0425 & 0.056 & 2.25 & 3.07 & 16.7 \\
\hline
\end{tabular}

TNF-alpha-inhibitor, HIF-alpha inhibitor, detoxicant (alcohol) antidote(aluminum)* $; 1.70 \%$ peak area was .gamma.-Sitosterol, it is PPAR -Gamma-Antagonist ${ }^{*}$ ( protection against obesity and related diseases such as type 2 diabetes $^{20} ; 1.44 \%$ peak area was $2,4 \mathrm{~A}, 8,8$ TETRAMETHYL-DECAHYDR CYCLOPROPA[D]NAPHTHALENE, it is Antimcrobial ${ }^{21}$. $2.37 \%$ peak area was Phytol, acetate, it is a Fragrance $22 ; 1.43 \%$ peak area was Oxirane, hexadecyl, whihx is an Antimicrobial ${ }^{24}$;

The proximate composition of Clerodendrum colebrookianum is recorded moisture as $77.90 \%, 4.28 \%$ carbohydrate, $2.36 \%$ crude protein, $4.21 \%$ crude fibre and $0.35 \%$ crude fat. The minerals concentrations are $0.215 \mathrm{mg}$ of $\mathrm{Fe} / \mathrm{g}, 0.105 \mathrm{mg} / \mathrm{g}$ of $\mathrm{Mn}, 0.0425 \mathrm{mg}$ of $\mathrm{Cu} / \mathrm{g}, 0.056 \mathrm{mg} \mathrm{Zn} / \mathrm{g}$, $2.55 \mathrm{mg}$ of Mangnesium/g, $4.3 \mathrm{mg}$ of $\mathrm{Na} / \mathrm{g}$ and $24.5 \mathrm{mg}$ of $\mathrm{K}$ per gram of sample.

\section{DISCUSSION}

Minerals and Proximate compositions in a food is vital for the proper growth and development of a healthy body and secondary metabolites included in diet act as a nutraceuticals thus help in fighting various health problems. Minerals contents and Proximate composition of Clerodendrum colebrookianum was recorded to be adequate for a healthy growth and development of a body and the secondary metabolite constituents was recorded to contain at least eleven compounds that are reported to be useful for high blood pressure problem. In addition, as many as twenty useful phytoconstutuents were recorded from the sample including antioxidant, anti uric acid formation, anti-tumour, help in zinc bioabiability and aromatic. The present study advocates the traditional knowledge on the use of Clerodendrum colebrookianum as a remedy for high blood pressure problem.

\section{ACKNOWLEDGEMENT}

The author highly grateful to the scientist in-charge CSIR-North East Institute of Science and Technology Branch, Itanagar, Naharlagun, Arunachal Pradesh, India and Principal, Jawaharlal Nehru College, Pasighat for providing laboratory facilities and Prof A.K.Das and Dr. R.Shankar for research guidance.

\section{REFERENCES}

1. King, H. "Let Food BeThy Medicine." Hippocrates Now: The 'Father of Medicine' in the Internet Age. London: Bloomsbury Academic. 2020;111-32.

2. Etkin, NL, Ross, PJ. Food as medicine and medicine as food. An adaptive framework for the interpretation of plant utilization among the Hausa of Northern Nigeria. Social Medicine. 1982;16:1559-73.

3. Pieroni, A, Price LL. Eating and Healing: Traditional Food as Medicine. 2006; Food Products Press, 10 Alice Street, Binghamton, New York. pp 1-3.

4. Raghavendra, N, Sneha, DB, Sulochana, B, Rabinarayan, A. Therapeutic potential of wild edible vegetables - A Review. Journal of Ayurveda and Integrated Medical sciences. 2(6):85-93.
5. Chanda,S, Tiwari, RK, Kumar, A, Singh, K. Nutraceuticals Inspiring the Current Therapy for Lifestyles Diseases. Advances in Pharmacological Sciences. 2019:1-5.

6. Radu, CF, Irina, F, Alina, O, Milen, IG, Elwira, S. Innovative Approaches for Recovery of Phytoconstituents from Medicinal/Aromatic Plants and Biotechnological Production: Molecules. 2020;25(2).

7. Cyrill, L, Gorlenko, HY, Kiselev, EV, Budanova, AA, Zamyatnin, Jr, Larisa, NI. Plant Secondary Metabolites in the Battle of Drugs and Drug-Resistant Bacteria: New Heroes or Worse Clones of Antibiotics? Antibiotics (Basel). 2020;9(4):170.

8. Akram, k, Bibi LH. Phytochemicals and Hypertension. Shiraz E Medical Journal. 2014;15(1).

9. Sabira, S, Hafiz, MA. Review: Medicinal Plants Combating Against Hypertension A Green Antihypertensive Approach. Pak J Pharm Sci. 2017;30(6):2311-9.

10. Oratai, N, Panadda, K, Kamonvadee, B. Medicinal plants used for hypertension treatment by folk healers in Songkhla province. Journal of Ethnopharmacology. 2018;58-70.

11. Nath, SC, Bordoloi, DN. Clerodendrum colebrookianum, a Folk Remedy for the Treatment of Hypertension in Northeastern India. Pharmaceutical Biology. 1991;29(2):127-9.

12. Namsa, ND, Mandal, M, Tangjang, S and Mandal, SC. Ethnobotany of the Monpa ethnic group at Arunachal Pradesh, India. Journal of Ethnobiology and Ethnomedicine. 2011;7:31.

13. Lokesh, D, Amitsankar, D. Evaluation of mechanism for antihypertensive action of Clerodendrum colebrookianum Walp., used by folklore healers in north-east India. J Ethnopharmacol. 2012;143(1):207-12.

14. Kanjilal, U and Borthakur, S. Flora of Assam; 1997. Vol. IV.Omsons Publications, New Delhi; (reprint). Pp-291-192.

15. https://pubchem.ncbi.nIm.nih.gov/compound/5 hydroxymethylfurfural\#section=IR-Spectra

16. Arora, S and Meena, S. GCMS profiling of Ceropegia bulbosaRoxb.var. bulbosa, an endangered plant from Thar Desert, Rajesthan. Th Pharma Innovation Journal. 2017:6(11):658-573.

17. Zih-Rou, H, Yin-Ku, Lin., and Jia-You, F. Biological and Pharmacological Activities of Squalene and Related Compounds: Potential Uses in Cosmetic Dermatology. Molecules. 2009;14:540-54.

18. Maryam, R, Behnaz, B, Mohammad, AS, Abdolrasoul, S, Seyed, JGH. Impact of Vitamin E Supplementation on Blood Pressure and Hs-CRP in Type 2 Diabetic Patients. Health Promot Perspect. 2012; 2(1):72-9.

19. Chandler, RF, Hooper, SN and Ismail, HA. Antihypercholesterolemic studies with sterols: beta-sitosterol and stigmasterol. J Pharm Sci. 1979;68(2):245-7.

20. Batta, AK, Xuab, G, Honda, A, Miyazaki, T, Salen, G. Stigmasterol reduces plasma cholesterol levels and inhibits hepatic synthesis and intestinal absorption in the rat. Journal of Pharmaceutical Sciences. 2006;55(3):292-9.

21. Kadowaki, T. PPAR gamma agonist and antagonist: Nihon Yakurigaku Zasshi. 2001;118(5):321-6.

22. Hasanain, KS, Haider, JM, Haider, MH and I, MH: Antibacterial Effect of Ginger (Zingiber officinale) Roscoe and Bioactive Chemical Analysis using Gas Chromatography Mass Spectrum: Oriental Journal of Chemistry. 2016;32(2):817-37.

23. FAOMHO Food Additive Evaluations (JECFA).

24. Aliyu, MM, Mohammed, Al, Abubakar, BA, Mikhail, SA, Nasir, TH, Ibrahim, AO. Chemical composition and antimicrobial activity of hexane leaf extract of Anisopus mannii (Asclepiadaceae). J Complement Med Res. 2015;4(2):129-33. 


\section{GRAPHICAL ABSTRACT}
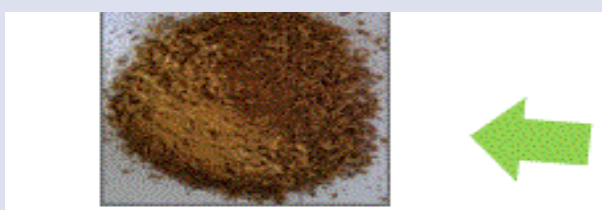

SHOOT POWDER

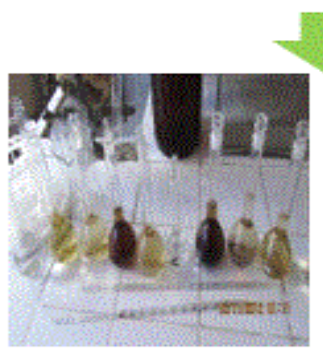

MINERALS STUDY

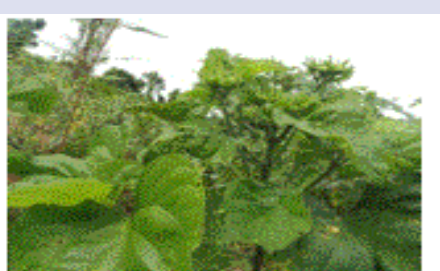

C. colebrookianum

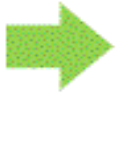

SHO
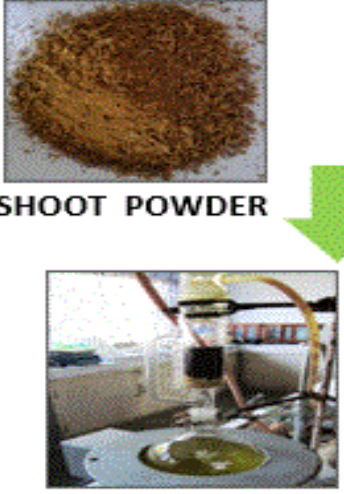

EXTRACTION

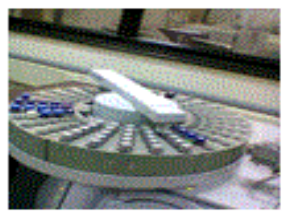

GCMS

dl-.alpha.-Tocopherol
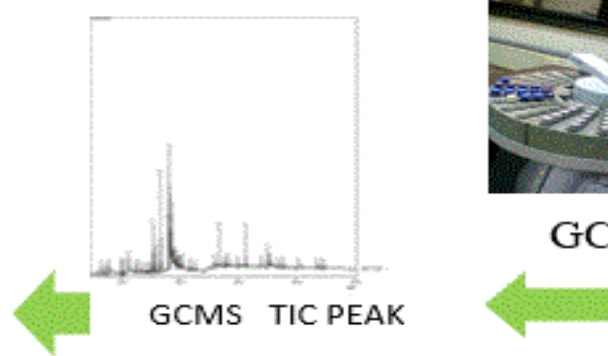

\section{SUMMARY}

- Clerodendrum colebrookianumWalp.is a medicinal food plant widely used for high blood pressure.

- Phytoconstituents and proximate were discoursed in the present study.

- Moisture content was recorded as $77.90 \%$, carbohydrate as $4.28 \%$, crude protein as $2.36 \%$, crude fibre as $4.21 \%$ and crude fat as $0.35 \%$ respectively. The minerals concentrations as $0.215 \mathrm{mg}$ of $\mathrm{Fe} / \mathrm{g}, 0.105 \mathrm{mg} / \mathrm{g}$ of $\mathrm{Mn}, 0.0425 \mathrm{mg} \mathrm{of} \mathrm{Cu} / \mathrm{g}$, $0.056 \mathrm{mg} \mathrm{Zn/g}, 2.55 \mathrm{mg}$ of Mangnesium/g, $4.3 \mathrm{mg}$ of $\mathrm{Na} / \mathrm{g}$ and $24.5 \mathrm{mg}$ of $\mathrm{K}$ per gram of sample.

- A total of eleven compounds are recorded to be useful for high blood pressure problem and as many as other twenty useful phytoconstutuents were recorded from the sample including antioxidant, anti-uric acid formation, anti-tumour, bioabiability of zinc etc.

- The present study advocates the traditional knowledge on the use of Clerodendrum colebrookianum as a remedy for high blood pressure problem.

\section{ABOUT AUTHORS}

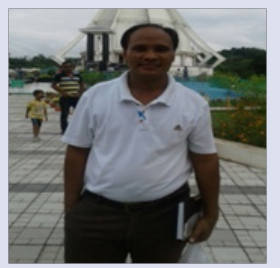

Temin Payum obtained his Ph.D degree in 2015 from Rajiv Gandhi University, Itanagar. Currently he is working as Assistant Professor at Jawaharlal Nehru College, Pasighat, Department of Botany, Arunachal Pradesh, India. His laboratory works on Ethnobotany, Antioxidants, Proximate, Anticancer herbs, Pharmacognosy and Phytochemisty on the medicinal food plants used among Tribal people of Arunachal Pradesh. He also works on natural detergent plants and formulation of personal care and kitchen care products based on natural plants.

Cite this article: Payum T. Phytoconstituents and Proximate Composition of Clerodendrum Colebrookianum Walp.: A Widely Used Anti High Blood Pressure Medicinal Food Plant in Eastern Himalayas. Pharmacogn J. 2020;12(6)Suppl:1534-40. 